\title{
An Amplitude Modulation/Demodulation Scheme for Whisker-Based Texture Perception
}

\author{
Yves Boubenec, ${ }^{1}$ Laure Nayelie Claverie, ${ }^{2,3}$ Daniel E. Shulz, ${ }^{1}$ and Georges Debrégeas ${ }^{2,3}$ \\ ${ }^{1}$ Unité de Neurosciences Information et Complexité (UPR 3293), Centre National de la Recherche Scientifique, 91198 Gif-sur-Yvette, France, ${ }^{2}$ Sorbonne \\ Universités, UPMC University of Paris 06, UMR 8237, Laboratoire Jean Perrin, F-75005, Paris, France, and ${ }^{3}$ CNRS, UMR 8237, Laboratoire Jean Perrin, \\ F-75005, Paris, France
}

Whisking rodents can discriminate finely textured objects using their vibrissae. The biomechanical and neural processes underlying such sensory tasks remain elusive. Here we combine the use of model micropatterned substrates and high-resolution videography of rats' whiskers during tactile exploration to study how texture information is mechanically encoded in the whisker motion. A biomechanical modeling of the whisker is developed, which yields quantitative predictions of the spectral and temporal characteristics of the observed whisker kinetics, for any given topography. These texture-induced whisker vibrations are then replayed via a multiwhisker stimulator while recording neuronal responses in the barrel field of the primary somatosensory cortex ( $1 \mathrm{bf}$ ). These results provide a comprehensive description of the transduction process at play during fine texture sensing in rats. They suggest that the sensory system operates through a vibratory amplitude modulation/demodulation scheme. Fine textural properties are encoded in the time-varying envelope of the whisker-resonant vibrations. This quantity is then recovered by neural demodulation, as it effectively drives the spiking-rate signal of a large fraction of $\mathrm{S} 1$ cortical neurons. This encoding/decoding scheme is shown to be robust against variations in exploratory conditions, such as the scanning speed or pad-to-substrate distance, thus allowing for reliable tactile discrimination in realistic conditions.

Key words: biomechanics; rat; resonance; somatosensory cortex; tactile; whiskers

\section{Introduction}

The tactile system of whisking rodents allows them to distinguish minute differences in surface texture. In the chain of transduction that leads to the sensory representation of the texture, the whisker plays an important role. This sensorless organ converts the physical properties of a surface into complex, time-varying mechanical stimuli that effectively drive the response of mechanoreceptors located in the whisker follicle. These peripheral neural signals are then processed along the somatosensory pathway, allowing the animal to probe some characteristics of the surface texture from which discrimination or identification tasks can be performed.

In 2003, two groups independently reported that the slender geometry of the whisker provides it with the properties of an under damped oscillator, which thus acts as a mechanical band-

Received Feb. 5, 2014; revised June 10, 2014; accepted June 15, 2014.

Author contributions: Y.B., D.E.S., and G.D. designed research; Y.B. performed research; Y.B., L.N.C., and G.D. analyzed data; Y.B., D.E.S., and G.D. wrote the paper.

This work was supported by CNRS, ANR (Project TRANSTACT 0294), and European Union 7th Framework Programme under grant agreement no. 269921 (BrainScaleS).

We thank L. Estebanez and S. El Boustami for their help with the electrophysiological recordings; V. Ego-Stengel and L. Bourdieu for fruitful discussions; P.J. Arduin, J.Y. Tiercelin, and P. Parra for technical help; and F. Petrelis and S. Neukirch for their invaluable contribution to the biomechanical model.

The authors declare no competing financial interests.

Correspondence should be addressed to Georges Debrégeas, Université Pierre et Marie Curie, Laboratoire Jean Perrin, 4 place Jussieu, 75005 Paris, France. E-mail: georges.debregeas@upmc.fr.

Y. Boubenec's present address: Laboratoire des Systèmes Perceptifs, CNRS UMR 8248, 29 rue d'Ulm, 75005, Paris, France.

DOI:10.1523/JNEUROSCI.0534-14.2014

Copyright $\odot 2014$ the authors $\quad 0270-6474 / 14 / 3410832-12 \$ 15.00 / 0$ pass filter (Neimark et al., 2003; Hartmann et al., 2003). Because of their various lengths, the whiskers' resonant frequencies span a wide range across the pad. This observation led Neimark et al. (2003) to propose a tonotopic scheme for texture encoding in which each whisker would transduce one particular spatial wavelength. This hypothesis, which made the whisker pad the equivalent of a tactile cochlea, failed to receive experimental confirmation at the neural level (Wolfe et al., 2008). Other studies have focused on the stick-slip instability, which is a ubiquitous phenomenon in solid friction. The rate of these rapid events varies with the surface granulometry, and could thus be central to the coding of texture information (Ritt et al., 2008; Wolfe et al., 2008; Lottem and Azouz, 2009).

Although these different studies have greatly improved our understanding of how the whisker mechanics and contact interaction shape the peripheral stimuli, a complete and comprehensive description of the whisker as an information transduction line is still lacking. One of the major and overlooked difficulties is that the information transduction operated by the whisker does not solely depend on its intrinsic geometrical and mechanical properties. It varies with contextual parameters, such as the position of the pad with respect to the object or the scanning speed, which can be actively controlled by the animal during exploratory tasks. A second major issue arises from the nature of the interaction between the physical world and the tactile organ. Because of the inherent nonlinearity of frictional interactions, the surface topography is converted into a sequence of forces acting at the whisker tip in a highly nontrivial fashion. 

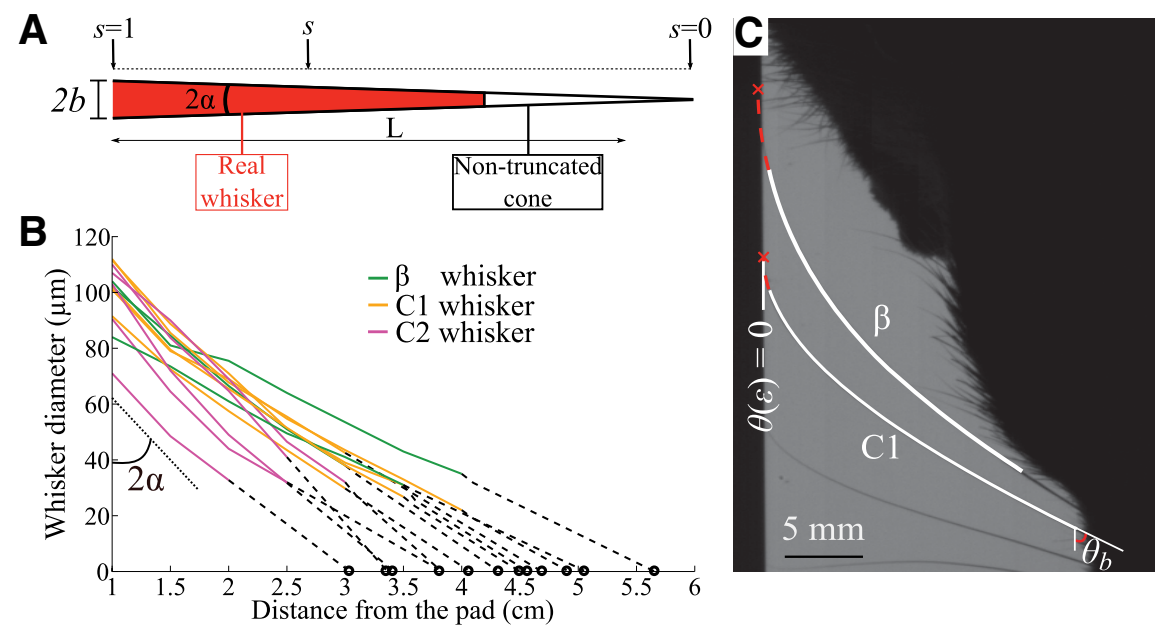

Figure 1. Whisker geometry and stationary profile during frictional contact. $A$, The whisker as a truncated tapered rod: definition of the curvilinear coordinate and geometrical parameters. $\boldsymbol{B}$, Whisker diameter measured as a function of the distance to the pad (solid lines) and linear extrapolation down to $s=0$ (dashed line) for different whiskers. The coordinate of the black circles (which corresponds to the cone tip) gives the nontruncated whisker length $L$. The cone angle $\alpha$ is extracted from the curve slope. C, Computed stationary whisker profiles (white lines) are shown for whiskers $\beta$ and C1. The angular profiles $\theta(s)$ are extrapolated to $\theta(\varepsilon)=0$ to extract the position $s=\varepsilon$ of the texture/whisker contact point along the whisker shaft (dashed red lines).

The present study addresses both aspects in the particular context of microtexture perception. Experiments were designed to optically record texture-induced whisker vibrations in headfixed anesthetized rats. These kinetic measurements were interpreted within the scope of a comprehensive biomechanical description of the texture-induced whisker dynamics, which takes into account: (1) the mechanical properties of the whisker, (2) the whisker/substrate configuration, and (3) a microscopic description of the whisker/texture frictional interaction. The neural processing operated by the somatosensory system was then investigated by recording the spiking response of cortical neurons in the S1 area to such mechanical stimuli.

\section{Materials and Methods}

\section{Probing texture-induced whisker vibrations}

Surgical preparation for in vivo experiments. Experiments were conducted in conformity with French (JO 87-848) and European legislation (86/ $609 / \mathrm{CEE})$ on animal experimentation. Adult male Wistar albino rats $(308 \pm 23 \mathrm{~g} ; N=6)$ were anesthetized with urethane $(1.5 \mathrm{~g} / \mathrm{kg}$, i.p.). Supplementary doses $(0.15 \mathrm{~g} / \mathrm{kg}$, i.p. $)$ were administered when necessary to maintain anesthesia, as assessed by the absence of corneal reflex, lack of response to mild hindpaw pinch, and lack of vibrissae movement. Atropine methyl nitrate $(0.3 \mathrm{mg} / \mathrm{kg}$, i.m. $)$ was injected to reduce respiratory secretions. Body temperature was maintained at $37^{\circ} \mathrm{C}$. The scalp was retracted after a subcutaneous injection of a local anesthetic (Lidocaine $1 \%)$. The surface of the skull was set horizontal. The skull was then cemented to a metal bar rigidly fixed to the experimental setup, which ensured recording stability across stimulation. The whiskers were trimmed off at the end of the experiments and their diameters were measured at different positions from base to tip.

Whisker stimulation by micropatterned surfaces. We systematically studied the kinetics of whiskers elicited upon moving planar textured substrates at constant speed along the animal. This work was limited to macrowhiskers ( $\beta, \mathrm{C} 1$, and $\mathrm{C} 2$ ), which are known to carry sufficient information for fine granulometry discrimination (Morita et al., 2011). All other whiskers were removed. The texture sample was attached via a $1 \mathrm{~m}$ long aluminum holder to a linear stepper motor (Linear Motor MLL302; Systro). An LED was positioned on the substrate holder to tag the video frame in which the whisker tip enters the textured region of the surface. A slab of similar thickness was juxtaposed to the stimulus substrate edge, preventing the large amplitude oscillations that otherwise may have resulted from the shock on the substrate edge. We checked for the absence of any measurable whisker oscillations before the tip entered the textured region of the substrate. The motor was controlled by an amplifier (EdiTask Software CNC; Systro) connected to a PC via a serial port. To minimize artifactual vibrations, the motor traveled on an air cushion and the whole apparatus was placed on antivibration rubber blocks. The range of scanning speeds $v_{\text {scan }}(100-200 \mathrm{~mm} / \mathrm{s})$ was chosen for its ethological relevance (Whishaw and Kolb, 2005). The anteroposterior axis of the animal head and the scanning axis were aligned. The pad-to-texture distance $H$ was varied by moving the frame onto which the animal was tethered relative to the texture (from 14 to $33 \mathrm{~mm}$ depending on the whisker), within a range consistent with natural exploration (Jenks et al., 2010). Each set of experimental conditions was tested with different textures exhibiting the same global statistics but different topographies (number of textures in each tested condition: 6-10). The presentation order was randomly shuffled from one experiment to the other.

The sample textures consisted of custommade $350 \times 40 \mathrm{~mm}$ printed circuit boards patterned with $1 \mathrm{D}$ random square-wave gratings $35 \mu \mathrm{m}$ in depth. The position of the grating edges were chosen from a Gaussian distribution. The mean spatial period of these barcode-like patterns was $0.6 \mathrm{~mm}$. The limit in feature width was imposed by the copper thickness (maximal lateral resolution: $35 \mu \mathrm{m}$ ). The maximum variation in distance from the sample axis to the head axis across the 35 $\mathrm{cm}$ long substrate was $400 \mu \mathrm{m}$ in the medial direction, yielding a maximum angle of divergence below $0.1^{\circ}$.

High-speed videography of whisker deflections. Whisker movements were recorded at a frame rate of $2 \mathrm{kHz}$ using a high-speed camera (Photron Fastcam SA3/105 mm f-2.8 DG Macro; Sigma; aperture time: 1/3000 s; pixel resolution: $95 \mu \mathrm{m}$, typical field of view: $500 \times 450$ pixels) mounted vertically above the animal. The whiskers were illuminated from below using a backlight (SSLUB, Phlox, and PP520; Gardasoft). The camera produced bird's-eye view movies of the whiskers. We chose to study whiskers $\beta, \mathrm{C} 1$, and $\mathrm{C} 2$ whose curvature plane are horizontal and can thus be entirely imaged with the optical configuration. We also wanted to capture whisker movements in the anteroposterior axis, since previous studies showed that most texture-elicited deformations occur along this axis (Arabzadeh et al., 2005; Lottem and Azouz, 2008). In most experiments, three whiskers appeared within the field of view (Fig. 1C). They were simultaneously tracked and their movements were analyzed independently.

Off-line whisker tracking. For each movie, different whiskers in contact with the texture were tracked using a semi-automated algorithm written in Python. We subtracted the average background image from each frame of the movie. Whisker centerline profiles were then extracted: we scanned all columns of each movie frame to locate the pixel of maximal intensity that defined an approximate position of the whisker. The whisker centerline in each column of the frame was defined as the barycenter of the five $(+2 /-2)$ pixels surrounding the pixel of maximal intensity (the pixels were weighted by their intensity), yielding a subpixel spatial resolution (noise level: $15 \mu \mathrm{m}=0.15$ pixel). The whisker dynamics were analyzed by monitoring the motion of equidistant fixed nodes positioned along the whisker shaft (median distance between nodes: $116 \mu \mathrm{m}=1.22$ pixels; 96 to $180 \mu \mathrm{m}$ depending on the experiment). Near the skin, the fur hampered proper observation of the whisker. We thus estimated the intersection between the whisker and the skin manually and linearly extrapolated the missing part. The back-illumination geometry combined with the bird's-eye optics resulted in shadowing $\sim 3.0 \mathrm{~mm}$ of the whisker near the substrate. The position of the whisker/texture contact point was thus determined by extrapolating the whisker profile (second 
degree polynomial; Fig. $1 C$, red dashed line). The contact location was taken as the point where the profile appears tangent to the substrate plane. In a few trials, when the contact point was too close to the physical tip of the whisker, the extrapolated contact position was clearly inconsistent with the substrate plane location. The corresponding trials were thus discarded in the analysis.

\section{Biomechanical model of the whisker}

The whisker as a tapered elastic beam. The biomechanical description of the whisker builds upon a model recently developed for a different context of exploration (Boubenec et al., 2012). Whiskers are described as slender tapered rods with no intrinsic curvature. We denote $L$ the length of the nontruncated cone, $2 b$ the whisker base diameter, and $\alpha=b / L$ the cone angle (Fig. 1A). The whisker profile is described using the curvilinear coordinate $s$ defined as the normalized arc length on the nontruncated whisker, such that $s=0$ and $s=1$ correspond to the cone tip and the whisker base locations, respectively.

From the measurements of the whisker diameter at different locations along its shaft, we extracted $L$ and $\alpha$ (Fig. $1 B$ ). Because of the truncation of the whiskers, $L$ can be significantly larger than the actual physical length of the whisker. For all whiskers, $\alpha$ was measured to be within a relatively narrow range, $1.510^{-3} \pm 4.10^{-4} \operatorname{rad}\left(<0.1^{\circ} ; N=13\right.$ whiskers $)$, as previously reported for rats and other whisker-bearing animals (Neimark et al., 2003; Williams and Kramer, 2010).

Equilibrium profile of a frictional whisker. Each imposed scanning condition corresponds to a unique whisker stationary profile, which was experimentally determined by averaging the instantaneous whisker profiles extracted throughout the entire sweep. These profiles are described in curvilinear angular coordinates $\theta=\theta(s)$, where $\theta=0$ defines the substrate axis. The spontaneous curvature and out-of-plane deformation are ignored, and we assume a constant frictional force at a single contact point of coordinate $s=\varepsilon$. Under these assumptions, the whisker stationary profile is expected to obey the differential equation (see Boubenec et al., 2012):

$$
\frac{\partial}{\partial s}\left(\frac{\left(s^{4} \theta^{\prime}\right)^{\prime}}{\cos (\phi-\theta+\theta(\varepsilon))}\right)=0,
$$

where $\phi$ is the friction angle (i.e., $\mu=\tan \phi$ is the dynamic friction coefficient). The localized contact assumption imposes the bending moment to be null at the contact point such that $\theta^{\prime}(\varepsilon)=0$. The whisker orientation $\theta(\varepsilon)$ at the contact point is set by the local slope of the scanned substrate. The whisker is assumed to be rigidly clamped in the follicle such that the base angle $\theta(1)=\theta_{b}$ is imposed by the pad configuration. With these three boundary conditions, Equation 1 can be numerically integrated and yields a unique solution for a given friction coefficient $\mu$. When computing the corresponding theoretical profiles, the results were found to weakly depend on the choice of the friction coefficient. We thus set this parameter to a typical value of $\mu=0.4$ (Persson, 2000). The comparison between experimental and computed profiles is shown in Figure 1C. A good match was obtained across whiskers and experiments $\left(R^{2}=0.80 \pm 0.29 ; N=623\right.$ trials $)$.

Whisker dynamics. The time evolution of the whisker profile during tactile exploration is characterized by the transverse displacement sequence $U(s, t)$, normalized by the nontruncated whisker length $L$. This adimensional function can be decomposed into a quasi-static (or stationary) component $U_{q s}(s, t)$ and a vibratory part $u(s, t)$. The latter is further decomposed along the eigenmodes $V_{i}(s)$, such that

$$
U(s, t)=U_{q s}(s, t)+u(s, t)=U_{q s}(s, t)+\sum_{i} q_{i}(t) V_{i}(s) .
$$

The resonant modes were numerically computed with boundary conditions $V_{i}(1)=V_{i}^{\prime}(1)=0$ (clamped at the whisker base), and $V_{i}(\varepsilon)=V_{i}^{\prime \prime}(\varepsilon)=0$ (pinned at the contact point). The latter condition reflects both the maintained frictional contact $\left(V_{i}(\varepsilon)=0\right)$ and the single contact point hypothesis, which imposes a null bending moment $\left(V_{i}^{\prime \prime}(\varepsilon)=0\right)$. Each mode is associated with a resonant angular frequency $\omega_{i}=\kappa_{i} / k$, where $\kappa_{i}$ is a numerical factor that only depends on $\varepsilon$, and $k$ is an intrinsic timescale characteristic of the whisker-free vibrations. It reads $k=2 \frac{L}{\alpha \sqrt{E / \rho}}$, where $E$ and $\rho$ are the whisker Young's modulus and density, respectively. Each resonant mode is associated with a harmonic equation of motion:

$$
\ddot{q}_{i}+2 \zeta \omega_{i} \dot{q}_{i}+\omega_{i}^{2} q_{i}=0,
$$

where $\zeta$ is a damping coefficient that accounts for all dissipative processes.

In the present study, the whisker oscillations were elicited by the frictional interaction between the whisker tip and a texture. The mode amplitude $q_{i}(t)$ thus obeys the forced oscillator equation:

$$
\ddot{q}_{i}+2 \zeta \omega_{i} \dot{q}_{i}+\omega_{i}^{2} q_{i}=f_{i}(t) .
$$

The driving signal $f_{i}(t)$ depends, in a nontrivial way, on the substrate topography and the whisker stationary configuration as detailed in the next section. To analyze the whisker vibratory spectra, we nevertheless assume that the spectrum of $f_{i}(t)$ reflects that of the topography. The use of a Gaussian white-noise texture thus allows us to predict that the power spectrum of each mode amplitude $q_{i}(t)$ can be fitted by a Lorentzian function:

$$
\left|\tilde{q}_{i}\right|^{2}(\omega) \approx \frac{1}{\left(2 \omega_{i} \zeta \omega\right)^{2}+\left(\omega^{2}-\omega_{i}^{2}\right)^{2}}
$$

Whisker response to a gap-crossing event. As demonstrated in Boubenec et al. (2012), the knowledge of the time evolution of the quasi-static profile $U_{q s}(s, t)$, allows one to derive the mode amplitude $q_{i}(t)$. Two situations must be considered.

(1) For a continuous evolution of $U_{q s}(t)$, the mode amplitude $q_{i}(t)$ reads:

$$
q_{i}(t)=-\int_{-\infty}^{t}\left[\int_{\varepsilon}^{1} s^{2} \ddot{U}_{q s}\left(s, t^{\prime}\right) V_{i}(s) d s\right] G\left(\omega_{i}, \zeta, t-t^{\prime}\right) d t^{\prime},
$$

where $G$ is the Green function of the dynamic system, i.e., the solution of the dynamic equation (4) to a Dirac of force:

$$
G\left(\omega_{i}, \zeta, t>0\right)=\frac{1}{\sqrt{1-\zeta^{2}} \omega_{i}} \sin \left(\sqrt{1-\zeta^{2}} \omega_{i} t\right) e^{-\zeta \omega_{i} t} .
$$

(2) If $U_{q s}(s, t)$ exhibits a discontinuity at $t=0$, then the solution reads, for $t>0$ :

$$
\begin{gathered}
q_{i}(t)=\left(\alpha_{i} \cos \left(\sqrt{1-\zeta^{2}} \omega_{i} t\right)+\beta_{i} \sin \left(\sqrt{1-\zeta^{2}} \omega_{i} t\right)\right) e^{-\zeta \omega_{i} t}, \\
\alpha_{i}=\int_{\varepsilon}^{1} s^{2} \Delta U_{q s}(s) V_{i}(s) d s \\
\beta_{i}=\frac{1}{\omega_{i}} \int_{\varepsilon}^{1} s^{2} \Delta \dot{U}_{q s}(s) V_{i}(s) d s
\end{gathered}
$$

where $\Delta U_{q s}=U_{q s}\left(0^{+}\right)-U_{q s}\left(0^{-}\right)$and $\Delta \dot{U}_{q s}=\dot{U}_{q s}\left(0^{+}\right)-\dot{U}_{q s}\left(0^{-}\right)$denote the discontinuity of the whisker displacement and velocity profiles at $t=0$, respectively.

This generic biomechanical description was used to compute the whisker dynamics elicited by active exploration of micropatterned substrates consisting of a series of gaps of varying width $\lambda$ and constant depth $h$. We first computed the dynamic response of the whisker elicited by the passage of one of these elementary topographical features. The sequence of vibrations induced by the complete topography was then obtained by summing these successive dynamic signals. The scanning speed $v_{\text {scan }}$, base angle $\theta_{b}$, and whisker base/substrate distance $H$ were assumed to be constant as was the case in the experiment (Fig. 2A). 

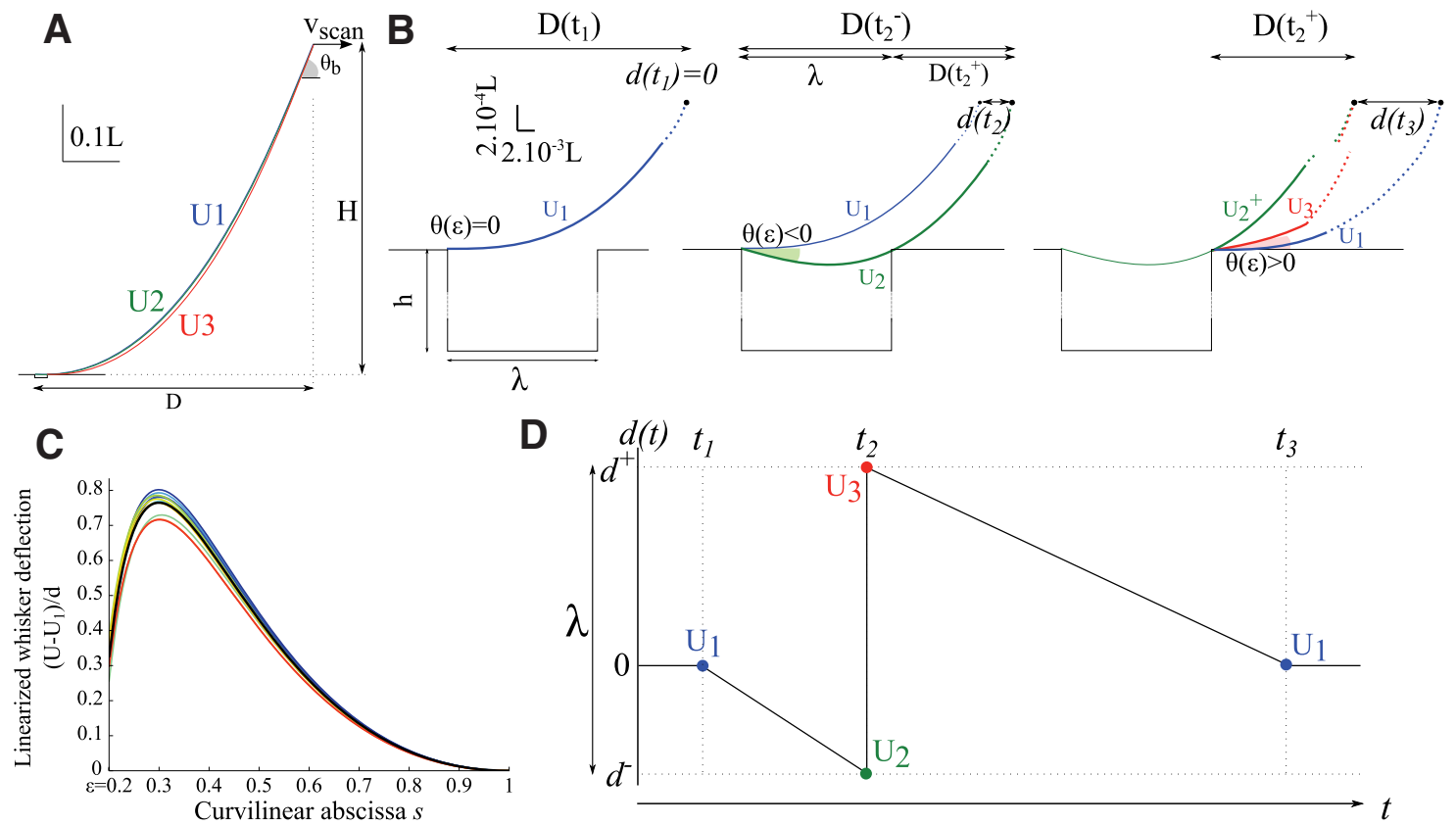

Figure 2. Evolution of the whisker quasi-static profile during a gap-crossing event. $A$, Definition of the geometrical parameters. The three intermediate profiles $U_{1}, U_{2}$, and $U_{3}$, are shown in blue, green, and red, respectively. $\boldsymbol{B}$, Close-up of the profiles at the whisker tip, showing the three successive phases (see text). The distance $d(t)$ denotes the variation of the contact to base distance $d(t)=D(t)-D\left(t_{1}\right)$. C, Variational quasi-static profiles normalized by $d:\left(U_{q s}(s, t)-U_{1}(s)\right) / d(t)$. The mean profile $\bar{U}$ is shown in black. $\boldsymbol{D}$, Time evolution of $d(t)$. The slope in the $\left[t_{1}, t_{2}\right]$ and $\left[t_{2}, t_{3}\right]$ intervals is $-v_{\text {scan }}$. The successive quasi-static configurations $U_{1}, U_{2}$, and $U_{3}$ are indicated on the graph.

We first derived the evolution of the quasi-static profiles $U_{q s}(s, \mathrm{t})$ during a gap-crossing sequence by solving Equation 1 throughout the sequence (Fig. 2B). The whisker initially scans the flat surface with its tip tangent to the surface. This initial profile can be obtained by numerically solving Equation 1 with boundary conditions $\theta(1)=\theta_{b}$ and $\theta(\varepsilon)=0$ over a wide range of values of $\varepsilon$. The quasi-static configuration, noted $U_{1}(s)$, corresponds to the unique solution that yields a whisker base/ substrate distance $H$.

At time $t=t_{1}$, the contact point reaches the first edge of the gap. It then continuously rolls around the edge. Notice that during this phase, the horizontal distance between the whisker base and the contact point increases linearly with time, $D(t)=D\left(t_{1}\right)+v_{\text {scan }}\left(t-t_{1}\right)$. Successive whisker profiles can be computed by solving the static equilibrium equation for $\theta(1)=\theta_{b}$ and various pairs of $(\varepsilon, \theta(\varepsilon))$. The configuration $U_{q s}(s)$ at time $t$, corresponds to the unique solution for which the horizontal and vertical distances between the whisker base and the contact are $D(t)$ and $H$, respectively. During this phase, the contact point location moves toward the whisker tip ( $\varepsilon$ decreases) and the angle at contact $\theta(\varepsilon)$ decreases.

At time $t=t_{2}$, the quasi-static profile intersects both edges of the gap. At $t>t_{2}$, the whisker rolls around the second edge. The procedure described above allows one to compute the further evolution of the quasi-static profile. Notice that the discrete change in the contact location produces a discontinuity in the quasi-static profile evolution at $t=$ $t_{2}$. At this time, the whisker configuration changes from $U_{2}(s)$ to $U_{3}(s)$. It then continuously evolves until recovering the initial configuration $U_{1}(s)$ at $t=t_{3}=\lambda / v_{\text {scan }}$.

Because of the small dimensions of the gaps with respect to the whisker length, the quasi-static configuration displays minute variations during this process, as shown in Figure $2 A$. This enables one to linearize the series of quasi-static profiles around the initial (and final) configuration $U_{1}(s)$, for a wide range of $\left[\theta_{b}, \varepsilon\right]$ (approximately $\theta_{b}<1.4$ and $\varepsilon>0.1$ ). Within this range, the gap-induced whisker deflection $U_{q s}(t)-U_{1}$ displays a quasi-linear dependence with the horizontal excursion of the contact point $d(t)=D(t)-D\left(t_{1}\right)$, such that the quasi-static deformation can be written as follows:

$$
U_{q s}(s, t)=U_{1}(s)+d(t) \bar{U}(s) .
$$

This is shown in Figure $2 C$ where the variational profiles $\left(U_{q s}(s, t)-U_{1}(s)\right) / d(t)$ are collapsed onto a single profile $\bar{U}(s)$, whose shape depends on the values imposed for $\theta_{b}$ and $H$. This linearization allows one to separate the time and spatial components of the quasi-static deformation sequence. The time evolution of $d(t)$ is shown in Figure $2 D$.

The variation in the contact point location $\varepsilon$ along the whisker shaft during a gap-crossing event is very limited (typically less than $0.02 \mathrm{~L}$ ). This quantity is therefore assumed to be constant when computing the eigenmodes and resonant frequencies. The mode decomposition procedure described in the preceding section can then be implemented yielding the full $U(s, t) d y-$ namics. Notice that the quasi-static evolution exhibits both a continuous modulation before and after $t_{2}$, and a discontinuity at $t=t_{2}$. The complete response of the whisker to a gap-crossing event can thus be written as follows: $r_{\lambda}(s, t)=d(t) \bar{U}(s)+\Sigma_{i}\left[q_{i}^{\text {kin }}(t)+q_{i}^{\text {elast }}(t)\right] V_{i}(s)$. The kinetic term $q_{i}^{\text {kin }}(t)$ is driven by the second time derivative of the quasi-static profile evolution and reads (see Eq. 6):

$$
\begin{array}{r}
q_{i}^{k i n}(t)=-v_{\text {scan }}\left(\int_{\varepsilon}^{1} s^{2} \bar{U}(s) V_{i}(s) d s\right)\left[G\left(\omega_{i}, \zeta, t-t_{1}\right)\right. \\
\left.-G\left(\omega_{i}, \zeta, t-t_{3}\right)\right] .
\end{array}
$$

The elastic term $q_{i}^{\text {elast }}(t)$ is driven by the sudden release of energy that results from the conformational change occurring at $t=t_{2}$. It is written as (see Eq. 8):

$q_{i}^{\text {elast }}(t)=\lambda\left(\int_{\varepsilon}^{1} s^{2} \bar{U}(s) V_{i}(s) d s\right) \cos \left(\sqrt{1-\zeta^{2}} \omega_{i}\left(t-t_{2}\right)\right) e^{-\zeta \omega_{i}\left(t-t_{2}\right)}$.

This approach allowed us, for most of the experimental conditions, to compute a predicted response $r_{\lambda}(s, t)$ of the whisker to the passage of an elementary gap of width $\lambda$.

Correlations between envelopes. The amplitude signals $q_{i}(t)$ for the three first modes were computed from both the experimental and predicted texture-induced whisker dynamics by projecting the dynamic 
term $u(s, t)$ onto the corresponding eigenmodes using the orthogonality property (see Boubenec et al., 2012 for a demonstration):

$$
q_{i}(t)=\int_{\varepsilon}^{1} u(s, t) s^{2} V_{i}(s) d s .
$$

The envelope of the whisker oscillations was then extracted using a Hilbert transform (Hill et al., 2011). When computing the cross-correlation of the two signals $q_{1,2}^{\text {pred }}(t)$ and $q_{1,2}^{\text {meas }}(t)$, we allowed for a small phase shift to account for the inherent uncertainty regarding the relative position of the pattern and the whisker tip. The time lag that produced the best correlation was found to be below $50 \mathrm{~ms}$.

\section{Electrophysiological recordings}

Surgical preparation for electrophysiological recordings. Experiments were performed in conformity with French (JO 87-848) and European legislation (86/609/CEE). Adult Male Wistar rats $(N=11$ rats, $302 \pm 15 \mathrm{~g})$ were deeply anesthetized (stage III, plane 3 ) with $3 \%$ isoflurane in 1 $\mathrm{L} / \mathrm{min} 80 \% \mathrm{~N}_{2} \mathrm{O}-20 \% \mathrm{O}_{2}$ and placed in a stereotaxic frame. The body temperature was maintained at $37^{\circ} \mathrm{C}$ with a feedback-controlled heating pad. The left posteromedial barrel subfield was exposed. Following surgery, isoflurane concentration was progressively adjusted $(2.5 \%-0.6 \%)$ to maintain the animal in a lightly anesthetized state (stage III, plane 1-2) based on three criteria: fast electrocorticogram $(\leq 5 \mathrm{~Hz})$ oscillations, a stable $1-1.5 \mathrm{~Hz}$ respiration rate, and a lack of spontaneous movements. After removing the dura, 32 channel silicon probes (NeuroNexus; recording size: $177 \mu \mathrm{m}, 8$ shank geometry) were inserted within the cortex. Spike sorting of single units was performed manually using Offline Sorter (Plexon) on the space defined by the first two principal components of the spike shapes. Spike clusters that were clearly separated from the noise cluster were isolated. They were retained as single units if the autocorrelogram displayed at least a $1 \mathrm{~ms}$ refractory period and the spike shape was "physiological" (biphasic) and consistent in time throughout the cluster. We allowed a limited drift of the spike shape amplitude during the recordings. We defined the signal-to-noise ratio (SNR) as the peak-to-peak amplitude of the smallest waveform for a given unit, divided by the rms of the noise. The above criteria yielded a set of units whose SNR ranged from 3.1 to 25 with $98 \%$ of all waveforms displaying a SNR above 4.0. Among them, the mean SNR was 6.7 and the median SNR was 6.2. We recorded 317 single units and 398 multi-units $(N=15$ recording sessions) and retained those with a mean firing rate above $1 \mathrm{~Hz}$ (143 single units, 289 multi-units), providing a sufficient number of spikes to perform a statistical analysis.

Multiwhisker stimulation. The 24 most caudal right whiskers of the rat were simultaneously and independently deflected in the rostrocaudal axis using a custom-built multiwhisker stimulator (Jacob et al., 2010). Whiskers were trimmed off at $10 \mathrm{~mm}$ from the pad and inserted $5 \mathrm{~mm}$ into each whisker stimulator. The resting angle was used as the stimulation zero position. The principal whisker of the recorded units was determined. Rat whiskers were deflected with a $10 \mathrm{~s}$ long sequence of whisker deflection applied to all whiskers and interlaced with other protocols that were not under the scope of this article. This sequence was temporally shifted from whisker to whisker, resulting in an uncorrelated stimulus across the whisker pad. The stimulus was presented $40-100$ times. The stimulus sequence was built from the whisker vibrations extracted by videography during tactile exploration of patterned substrates. We used recordings done with three different textures in an anesthetized rat $\left(\beta\right.$ whisker, $\varepsilon=0.29, v_{\text {scan }}=100 \mathrm{~mm} / \mathrm{s}$ ). We extracted the movement of the whisker at $5 \mathrm{~mm}$ from its base. The stimulus signal was Bessel filtered to match the $0.1-83 \mathrm{~Hz}$ bandwidth of the whisker stimulator. The stimulus was well reproduced by the piezoelectric actuators, as checked by measuring the level of correlation between the command and the actual motion $(r=0.991)$ with a laser telemeter (Micro-Epsilon). No noticeable ringing was present in the actuator motion.

Analysis of electrophysiological data. The envelope $A(t)$ of the mechanical stimulus was extracted using the Hilbert transform (Hill et al., 2011). The firing rate was averaged across trials. We discretized the average firing rates and the envelope of the stimulus with $10 \mathrm{~ms}$ bins. We then calculated the cross-correlation between the average firing rate and the stimulus envelope. We also computed the cross-correlation between shuffled firing rates and the stimulus envelope (300 times for each singleunit and multi-unit recording). A threshold of significance was defined as the 99th percentile of the distribution of all 300 cross-correlograms. The correlation between the stimulus envelope and the observed firing rate was considered statistically significant when the cross-correlation exhibited a central peak that exceeded the threshold of significance. Firing rate signal was allowed to be shifted by \pm 1 bin to maximize the correlation between the stimulus envelope and the firing rate.

\section{Analysis and statistical methods}

All analyses were done in MATLAB 7.6.0 (MathWorks). Numerical integration was performed using Mathematica (v8.0; Wolfram Research). Average data is expressed as mean \pm SD unless stated otherwise. Error bars in figures show the SD. All data distributions were examined for normality using Shapiro-Wilk (for $N<30$ ) or Kolmogorov-Smirnov (for $N>30$ ) tests. The significance of differences was set at $\alpha=0.05$ unless stated otherwise. Statistical significance was evaluated using Kolmogorov-Smirnov, ANOVA, and Kruskal-Wallis tests. When multiple groups were used, significant pairwise differences were determined using post hoc Tukey's test. Correlation was assessed with linear Pearson correlation.

\section{Results}

\section{D substrates elicit deterministic whisker motion}

The experimental setup mimicked the conditions of frictional contact imposed on whiskers during walking or trotting locomotion in freely moving animals (Whishaw and Kolb, 2005). Micropatterned substrates were scanned across the whiskers of anesthetized rats $\left(N_{\text {animal }}=6 ; N_{\text {whisker }}=13\right)$ at constant scanning speed $v_{\text {scan }}$ and pad-to-texture distance $H$ (Fig. $3 A$ ). Whisker movements were optically tracked with a high-speed camera and their centerline profiles were then extracted within each frame (Fig. 3B, white lines). The whisker oscillatory motion was further analyzed by monitoring the motion of equidistant nodes placed along the whisker centerline.

The trajectories of the nodes are essentially normal to the time-averaged profile with a maximum oscillatory amplitude of the order of a few tens of micrometers (Fig. 3C). The use of 1D micropatterned substrates, previously used to investigate texture transduction in human digital touch (Scheibert et al., 2009), results in highly reproducible texture-evoked whisker oscillations. This was established by repeating the experiments $\left(N_{\text {whisker }}=4\right.$; five runs each) using the same texture, pad-to-texture distance, and scanning speed. Figure $3 D$ shows, as an example, five successive recordings of the motion of one node on a $\mathrm{C} 2$ whisker as it is scanned across the same portion of a texture at $200 \mathrm{~mm} / \mathrm{s}$. The different traces appear almost identical from one run to the next, as confirmed by the strong correlation $(\rho=0.96 \pm 0.05)$ observed between individual traces and the five runs average (Fig. $3 E$ ).

Although there is no obvious relationship between the stimulus pattern and the whisker vibrations sequence, each texture and set of scanning conditions produces a deterministic signal. The high level of reproducibility between runs constitutes a major asset of these types of substrates compared with sandpaper samples (Ritt et al., 2008; Wolfe et al., 2008; Lottem and Azouz, 2009), with which any minute change in the contact point trajectory may induce significant modifications of the whisker dynamics on a trial-by-trial basis.

\section{Modal analysis of the texture-induced whisker vibrations}

The texture-induced transverse vibrations were obtained by subtracting the time-averaged whisker deformation from the instantaneous profiles extracted from each frame (Fig. 4A). These profiles were then normalized by the length $L$ of the nontrun- 

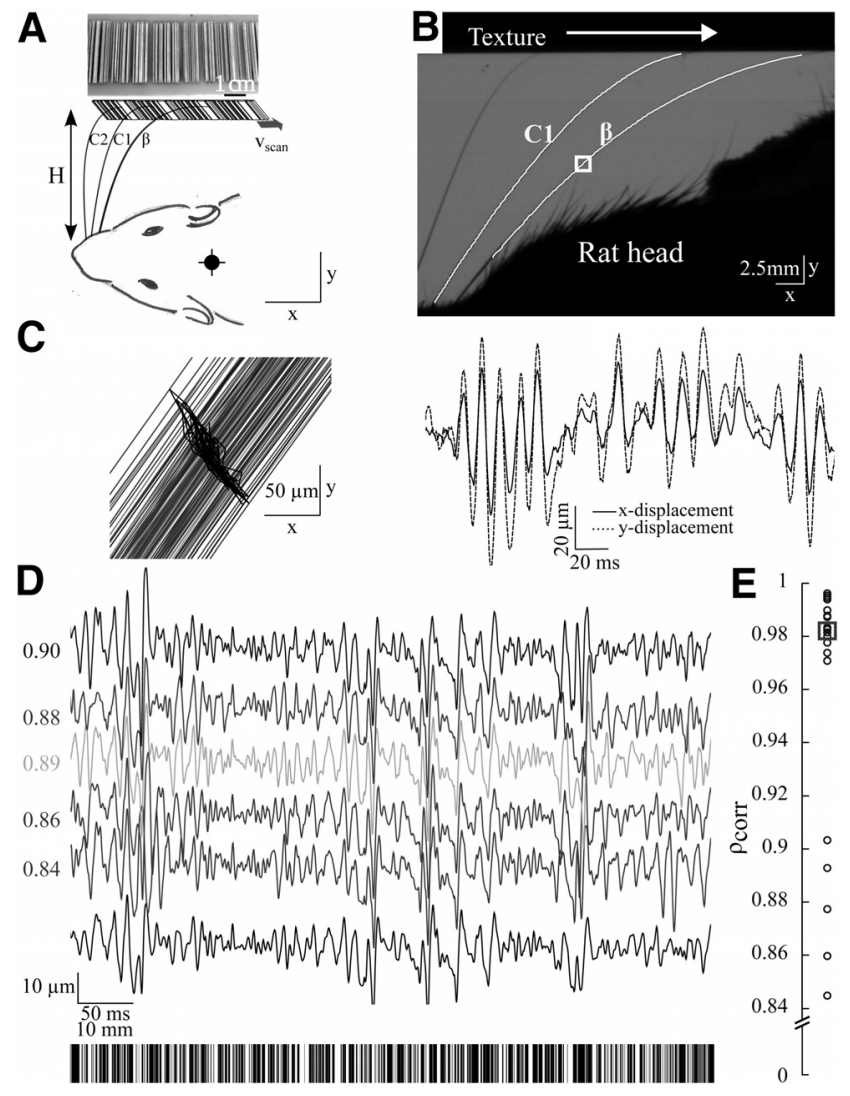

Figure 3. Video-tracking of texture-induced whisker vibrations. $A$, Sketch of the experimental setup. A white-noise texture (1D random square-wave grating) is mounted onto a linear motorized stage moving at a fixed scanning velocity $v_{\text {scan. }}$. The animal is positioned at a controlled pad-to-texture distance $H$. A bird's-eye view camera records images of the whiskers swept across the texture. $\boldsymbol{B}$, Example frame taken from a movie. The white line is the result of the whisker tracking for whiskers $\beta$ and $C 1$. The $(2$ whisker is out of focus and cannot be tracked. C, Deterministic motion of the whisker. Left, The region shown is the magnification of the white square in $\boldsymbol{B}$. Each colored trace shows a blow-up of the instantaneous whisker profile extracted from one frame. The black trace is a single node trajectory. Right, $x$-and $y$-displacement of the node highlighted on the left side. $\boldsymbol{D}, y$-displacement of a single node tracked on whisker $C 2$ for several runs under identical scanning conditions (texture topography, scanning speed, and radial distance). The various traces correspond to the node motion as the whisker tip scans the same region of the texture (shown at bottom). The number on the left of each trace is the correlation factor computed between the run and the motion averaged across the five successive runs (black trace at the bottom). $\boldsymbol{E}$, Correlation factors measured between each run and the average motion ( $N=4$ experiments; 5 runs each). The square indicates the median value of the dataset.

cated whisker (measured postmortem), yielding the adimensional vibratory signal $u(s, t)$. To quantitatively analyze the spectral characteristics of the frictional whisker, $u(s, t)$ was decomposed along the resonant eigenmodes $V_{i}(s)$ following the approach developed previously (Boubenec et al., 2012):

$$
u(s, t)=\sum_{i} q_{i}(t) V_{i}(s) .
$$

The first three eigenmodes $V_{i}(s)$ and frequencies $\omega_{i}$ were numerically computed using a set of boundary conditions imposed by the model hypothesis (clamped at the follicle, pinned at the contact point $\mathrm{s}=$ $\varepsilon)$. The amplitude $q_{i}(t)$ of each mode was then extracted by projecting Equation 15 onto $V_{i}(s)$ (see Materials and Methods).

This mode decomposition is illustrated in Figure $4 B$, which shows for a given time $t$ the instantaneous profile $u(s, t)$ together with each component $q_{i}(t) V_{i}(s), i=1-3$ and with the sum of the three modes $\sum_{i=1}^{3} q_{i}(t) V_{i}(s)$. The profile $u(s, t)$ displays a great similarity of shape with the fundamental eigenmode $V_{1}(s)$, which
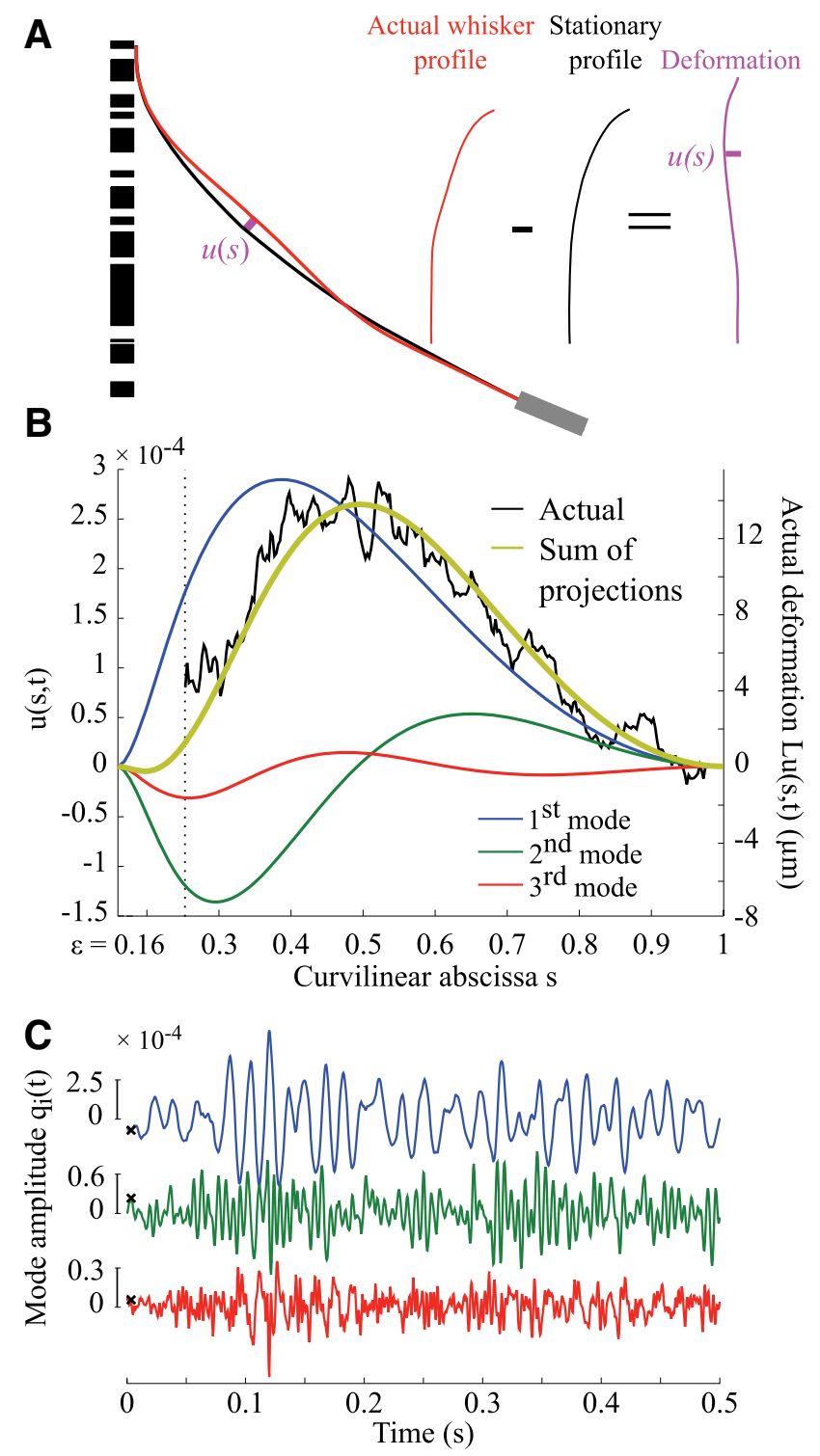

Figure 4. Modal analysis of the whisker kinetics. $\boldsymbol{A}$, The texture-induced deformation $u(s, t)$ (pink line) is obtained by subtracting the instantaneous (red line) and the stationary (black line) whisker profiles. $\boldsymbol{B}$, Mode decomposition of the texture-induced whisker deformation $u(s, t)$ (shown in black, whisker $\beta$, speed: $100 \mathrm{~mm} / \mathrm{s}$ ). Each mode component $q_{i}(t) V_{i}(s)$ and the sum of the three first modes $\Sigma_{i=1}^{3} q_{i}(t) V_{i}(s)$ are superimposed. The right axis indicates the actual deformation $L u(s, t)$ in micrometers. $C$, Excerpt of the mode amplitudes $q_{i}(t)$ of the three first modes. The black crosses correspond to the particular configuration shown in $\boldsymbol{B}$.

indicates that the whisker kinetics is dominated by the first resonant mode of oscillations. Across the runs, the first mode alone accounts for $66.4 \% \pm 10.7(N=582$ sweeps, $3500-7000$ frames/ sweep) of the variance of the displacement profiles. The 2 nd and the $3 \mathrm{rd}$ modes additionally account for $18.6 \% \pm 5.6$ and $5.6 \% \pm$ 2.9 of the variance (variance explained by 1 st $+2 \mathrm{nd}+3 \mathrm{rd}$ modes: $90.6 \% \pm 6.1$ ). When repeated across the entire sweep, this procedure yielded three time series $q_{1-3}(t)$ as shown in Figure $4 C$.

Because of the Gaussian white noise characteristics of the scanned topography, the spectrum of each of these signals is expected to display a Lorentzian shape (see Materials and Methods). This form consistently captures the measured spectra of $q_{i}$ (Fig. $5 A ; 1$ st mode: $R^{2}=0.55 ; 2$ nd mode; $R^{2}=0.73 ; 3$ rd mode: $\left.R^{2}=0.73\right)$. Although the 1 st (dominant) mode tends to contaminate higher harmonics, the mode-decomposition approach allows 
one to separate the successive resonant peaks. This clear separation proves impossible on the raw data as shown in Figure 5B, which displays the spectrum computed on the raw $y$-displacement of a single node. Here, the peaks associated with the 2 nd and 3rd modes barely emerge from the noise baseline.

From the Lorentzian fit, the resonance frequency $\omega_{i}$ and damping factor $\zeta$ were extracted for each mode (Fig. $5 A$ ). This process was repeated for all experimental configurations (1st mode: $R^{2}=0.63 \pm$ 0.19; 2nd mode: $R^{2}=0.70 \pm 0.14, N=$ 582). Although the 3rd mode is always present, its relatively small amplitude results in a large uncertainty in its frequency. The following analysis thus focuses on the first two modes.

Throughout the experiments, the value of the damping factor was found to fall within a narrow range (1st mode: $\zeta=0.11 \pm 0.05$; 2nd mode: $\zeta=0.13 \pm 0.05, N=582$ ) consistent with values previously reported in isolated whiskers (Hartmann et al., 2003; Neimark et al., 2003; Boubenec et al., 2012). The resonant frequencies of both modes were found to increase as the contact position was moved toward the whisker follicle, i.e., for increasing $\varepsilon$, as shown in Figure $5 B$. They are independent of the scanning speed for both the 1st mode (speed: $p=0.64, \varepsilon: p<10^{-15}$ ) and the 2nd mode (speed: $p=0.56, \varepsilon: p<$ $\left.10^{-15}\right)$, but vary significantly with the whisker identity and among animals, reflecting the variability of the whisker length and base diameter.

These observations are in quantitative agreement with the biomechanical model of the frictional whisker (see Materials and Methods). The latter predicts that the resonant frequency can be written as $\omega_{i}$ $=\kappa_{i}(\varepsilon) / k$, where $\kappa_{i}$ is an adimensional function of the contact point location $\varepsilon$ and $k=2 \sqrt{\rho / E} L / \alpha$ is a characteristic resonant period that depends on intrinsic biomechanical and geometrical parameters. The measured frequencies can thus be collapsed onto a universal curve by plotting the adimensional frequency $\kappa_{i}=k \omega_{i}$ as a function of $\varepsilon$ (Fig. 5C). The timescale $k$ was computed for each whisker using the measured values of $L$ and $\alpha$, and the value of $\rho$ previously reported ( $\rho=$ $1.14 \times 10^{3} \mathrm{~kg} / \mathrm{m}^{3}$; Hartmann et al., 2003). The Young modulus $E$ was chosen such as to provide a consistent fit with the computed values of $\kappa_{i}(\varepsilon)$. The best match between experimental and numerical adimensional frequencies (1st mode: $R^{2}=0.98, N=502$; 2nd mode: $R^{2}=0.97, N=523$ ) was obtained for $E=3.74 \mathrm{GPa}$, a value consistent with the existing literature $[E=3.68 \mathrm{GPa}(3-4$ GPa; Hartmann et al., 2003; 3.5 GPa; Solomon and Hartmann, 2006; 3-6 GPa; Birdwell et al., 2007)]. This agreement was maintained across modes and experiments (in all cases, $R^{2}>0.8$ ). freely oscillating whisker.
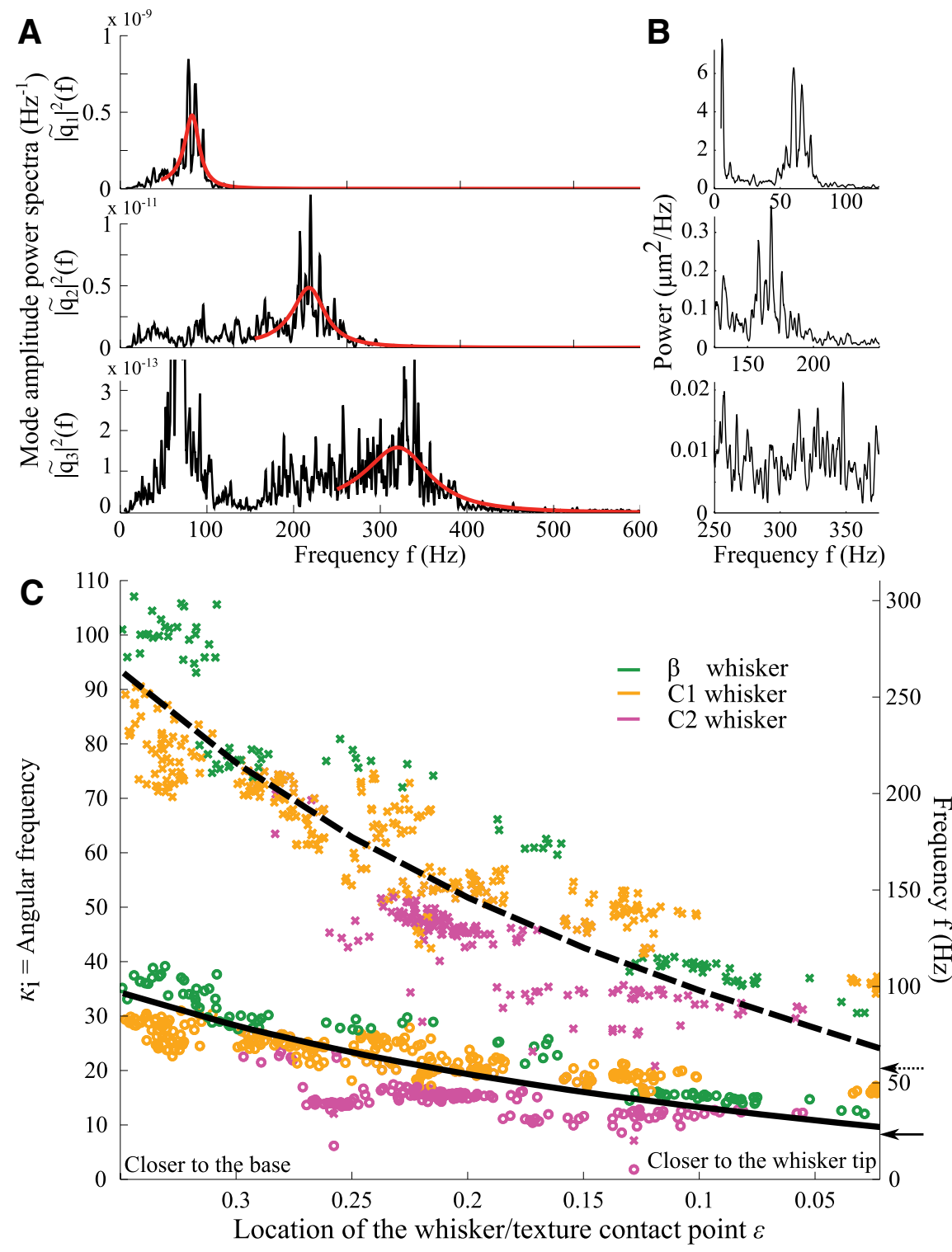

Figure 5. Resonance characteristics of the frictional whiskers. A, Power spectra of the amplitude signal for the three first modes (black line; from top to bottom, 1st, 2nd, and 3rd mode). These data correspond to the same trial as in Figure 4. The spectra de on the whisker, with close-ups around the three resonant frequencies. $($. Adimensional angular frequency $\kappa=k \omega$ for 1 st . for an average $\left(1\right.$ whisker are shown on the right axis. The arrows further indicate $\kappa_{1}^{\text {air }}$ and $\kappa_{2}^{\text {air }}$, as computed for an isolated

Notice, however, that a minor whisker dependence of the adimensional resonant frequencies (consistent for both modes) persists, which might be the signature of a slight dependence of the Young modulus with the whisker identity (Quist et al., 2011).

These results establish the validity of the biomechanical model of resonance in contacting whiskers, and in particular the pinned boundary conditions imposed at the contact point. They demonstrate that the whisker resonance characteristics during actual exploratory phases can be actively tuned by the animal through a unique contextual parameter, which is the location of the contact point along the whisker shaft. For a given whisker, the effective resonant frequencies, relevant to tactile sensing, appear significantly higher (up to a factor of 4 within the pad-to-texture distances explored in the present study) than the free vibration 

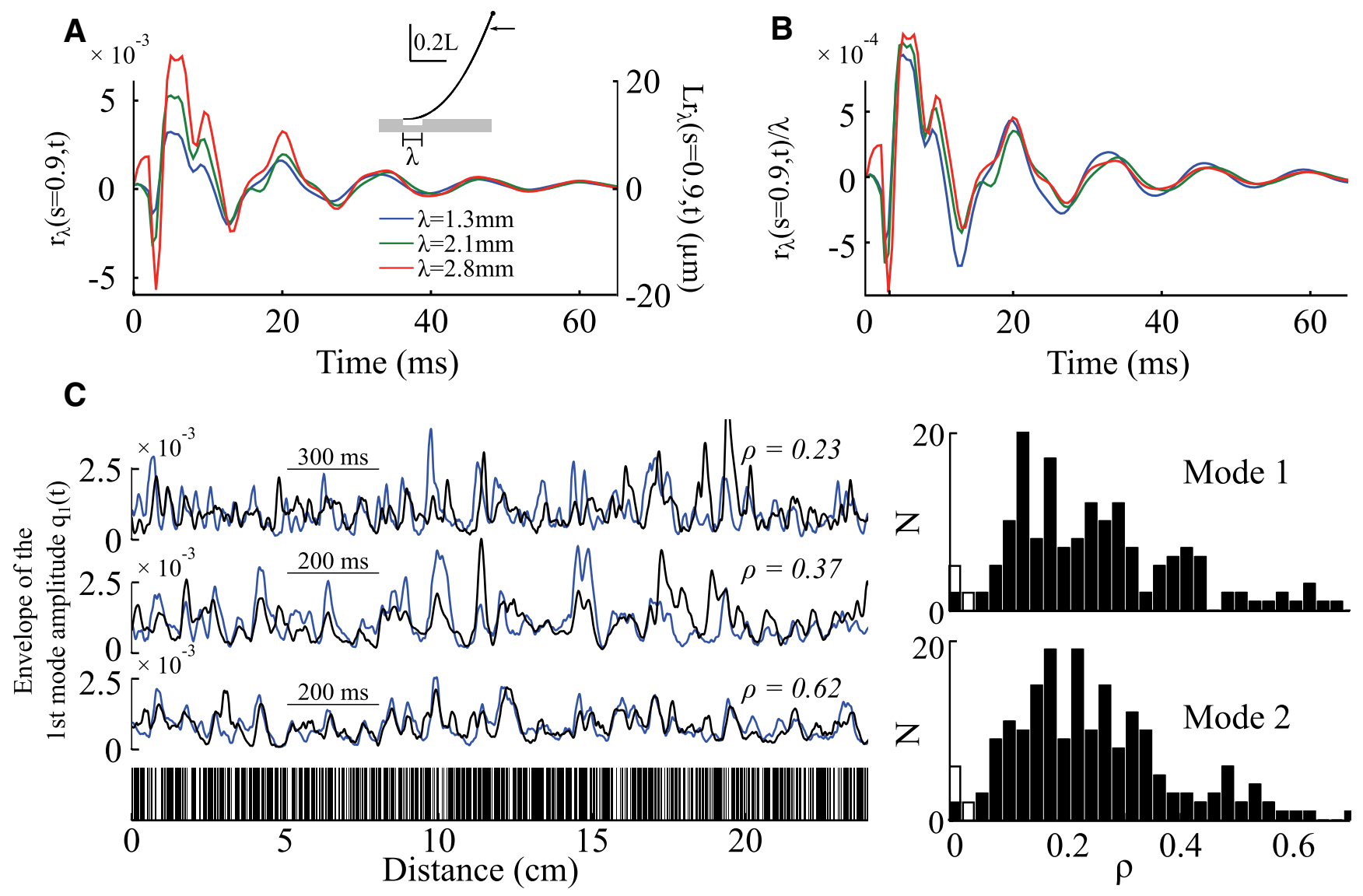

Figure 6. Predictions of texture-induced oscillations. $A$, Computed vibratory responses of a whisker to the passage of a rectangular trough of width $\lambda$. The transverse displacement of a whisker node at $s=0.9$ (i.e., at a distance $0.1 \mathrm{~L}$ from the whisker pad) is plotted for different values of $\lambda$. The time $t=0$ corresponds to the whisker tip reaching the first edge of the trough. The whisker displacement is shown in units of $L$ and in micrometers (for $L=40 \mathrm{~mm}$ ). Inset, Complete whisker profile at $t=0$. The arrow indicates the position of the node. $\boldsymbol{B}$, Transverse displacement normalized by the trough width $\lambda$, for the different values of $\lambda$. $\boldsymbol{C}$, Three examples of measured (black traces) and predicted (blue traces) envelopes of the first mode oscillation as a function of the scanned distance. The corresponding texture for the three times series is shown below. The three plots were obtained with the same whisker, same animal, and same texture but for different exploratory parameters, yielding three different correlation values as indicated. The predicted signal is normalized by the mean amplitude of the measured signal. $\boldsymbol{D}$, Distribution of correlation values between predicted and measured 1st- and 2nd-mode envelopes. White bars correspond to nonsignificant correlation values.

frequencies measured in isolated whiskers. The latter are indicated by the arrows on Figure $5 C$ for comparison $\left(\kappa_{1}^{\text {air }}=8.72\right.$, $\kappa_{2}^{\text {air }}=21.15$; Hartmann et al, 2003, their Table 1 ).

The mode decomposition approach allowed us to separate the spatial and temporal components of the texture-induced whisker kinetics. They are characterized by the series of eigenmodes $V_{i}(s)$ and adimensional mode amplitudes $q_{i}(t)$, respectively. In the rest of the article, we investigate the dependence of $q_{i}(t)$ with both the substrate topography and exploratory context. However, one should be aware that the whisker kinetics along its entire shaft can be recovered from the knowledge of $\left\{V_{i}(s), q_{i}(t)\right\}$ using Equation 15. In particular, one can derive in a straightforward way any effective stimulus signal in the follicle such as the whisker base curvature, moment, force, or any of their time derivatives. The whisker base curvature, for instance, reads:

$$
C(t)=\left.\frac{1}{L} \frac{\partial^{2} u(s, t)}{\partial s^{2}}\right|_{s=1}=\frac{1}{L} \sum_{i} q_{i}(t) V_{i}^{\prime \prime}(s=1) .
$$

The texture topography shapes the envelope of whisker oscillations

The amplitudes $q_{i}(t)$ of the resonant modes have the characteristics of sharply tuned oscillating signals of frequency $\omega_{i}$, with timevarying amplitude $A_{i}(t)$ and phase $\phi_{i}(t)$ (Fig. $\left.4 C\right)$ :

$$
q_{i}(t)=A_{i}(t) \cos \left(\omega_{i} t+\phi_{i}(t)\right) .
$$

We now investigate how the topography of the scanned substrates shapes the slowly varying amplitude signal $A_{i}(t)$. A mechanistic model, detailed in the Materials and Methods section, was developed, which predicts the whisker oscillations elicited by the frictional interaction between the whisker tip and the surface pattern. The topography $T(x)$ of the surface is described as a series of $N$ individual rectangular troughs such that:

$$
T(x)=\sum_{j=1}^{N} H\left(x-x_{j}, \lambda_{j}\right),
$$

where the series $\left\{x_{j}, \lambda_{j}\right\}_{j=1, ., N}$ defines the positions and widths of the troughs. In this expression, $H(x, \lambda)$ is a step function of width $\lambda$ whose first edge is located at $x=0$. The first step consists in deriving the quasi-static evolution of the whisker as its tip passes across such an isolated trough. This quasi-static sequence is then used to compute the whisker oscillatory signal $r_{\lambda}(s, t)$ elicited by the passage of the trough.

Figure $6 \mathrm{~A}$ shows the whisker motion evaluated near the follicle $r_{\lambda}(s=0.9, t)$ for different values of the trough width $\lambda$. All parameters were extracted from the stationary profiles $\left(\varepsilon\right.$ and $\left.\theta_{b}\right)$ and the measured vibratory spectra characteristics $\left(\omega_{i}\right.$ and $\left.\zeta\right)$. These response signals are characterized by a bout of oscillations 
decaying over a time period $\left(\omega_{i} \zeta\right)^{-1}$. The maximum amplitude grows quasi-linearly with $\lambda$ as shown by the collapse of the three signals when normalized by $\lambda$ (Fig. 6B). For a given topography, a complete prediction of the texture-induced whisker dynamics can thus be written as follows:

$$
R^{\text {pred }}(s, t)=\sum_{j=1}^{N} r_{\lambda_{j}}\left(s, x_{j} / v_{s c a n}\right) .
$$

To compare the predicted and measured whisker dynamics, we focused on the envelopes of the two first mode amplitudes, noted $q_{1,2}^{\text {pred }}(t)$ and $q_{1,2}^{\text {meas }}(t)$. Figure $6 C$ shows the best adjustment of predicted and measured envelopes (first mode) for three runs with the same whisker and the same texture, at different speeds and contact point locations (see Materials and Methods). Throughout the runs, the correlation values were found to vary over a wide range $(\rho=0.25 \pm 0.15 ; N=165$; Fig. $6 D)$. However, $98 \%$ of the predicted signals exhibit a significant correlation with their experimental counterpart (161/165). The large dispersion of the correlation values does not reflect a systematic dependence with any of the experimental parameters $\left(\varepsilon: p=0.95 ; \theta_{b}: p=0.96\right.$; whisker identity: $p=0.40 ; N=165$ ). These observations hold true for the 2 nd mode with $97 \%$ of predicted signals (186/192; $\rho=0.24 \pm 0.14 ; N=192$; Fig. $6 D$ )

The frictional/vibrational model of texture transduction correctly captures the time evolution of the whisker oscillatory dynamics. It validates the computed whisker response to a single trough. In this elementary process, the response is mostly controlled by the whisker contact with the second edge of the gap. This conformational change triggers a bout of vibration whose amplitude is set by the gap width. This dependence is consistent with the experimental observation that larger gaps induce larger whisker vibrations. The relatively low value of the mean correlation between experiments and theoretical predictions can be understood when considering that the successive gaps elicit whisker oscillations whose interference can be either constructive or destructive. The amplitude of the whisker oscillations thus critically depends on any phase shift that can result from minute in-plane displacement of the whisker tip during scanning.

Each substrate drives the whisker oscillations in a texturespecific way, but the amplitude of these oscillations depends on the context of exploration, i.e., the scanning speed $v_{\text {scan }}$, the base angle $\theta_{b}$, and the location of the point of contact $\varepsilon$. In the rest of this section, we thus systematically examine how these three different parameters control the transduction gain defined as the rms of the fundamental mode amplitude.

The first observation is that the amplitude of the textureinduced vibrations shows no significant dependence with the scanning speed $v_{\text {scan }}(p=0.11 ; N=582 ;$ Fig. $7 A-C)$. In contrast, the transduction gain was found to greatly vary with the base angle $\theta_{b}$, and, to a lesser extent, with the location of the contact point $\varepsilon$ along the whisker shaft $\left(\varepsilon: p<10^{-6} ; \theta_{b}: p<10^{-15}\right.$; Fig. $7 A, D)$. More specifically, the gain increases by up to a factor of two when the base angle increases from 0.8 to $1.4 \mathrm{rad}\left(45\right.$ to $\left.80^{\circ}\right)$. The gain slightly increases with decreasing $\varepsilon$, although this dependence is only significant for a large base angle. Due to the morphology of the whisker pad, the large angle data points are principally associated with the $\mathrm{C} 2$ whisker. We thus tested whether the dependence on $\theta_{b}$ did not merely reflect a whiskerspecific mechanism, by restricting the statistical analysis to $\beta$ and $\mathrm{C} 1$ whiskers. Even for these two whiskers, the $\theta_{b}$ dependence turns out to be highly significant $\left(\theta_{b}: p<10^{-15} ; N=360\right)$.
The same analysis was performed on the theoretical signals. Consistent with the experimental observations, the scanning speed was found to marginally impact the amplitude of the whisker oscillations. The dependence of the transduction gain with the two other contextual parameters (Figure $7 D$ ) is also correctly captured by the biomechanical model (Fig. 7E). The gain increases with increasing base angle, while showing a modest increase with decreasing $\varepsilon$ in the large base angle configuration. Notice, however, that the range of variations is larger in the model than in the experiment, which results in an overall overestimation of the predicted oscillation signal at large $\theta_{b}$. This discrepancy may result from the fact that in this large base angle regime, the system significantly departs from the small deformation limit used to compute the whisker mechanical response.

\section{The cortical response is driven by the envelope of the whisker oscillatory dynamics}

The previous sections helped clarify how a particular surface pattern, within a given exploratory context, is encoded at a preneuronal level in the envelope of the whisker resonant oscillations. We now turn to investigate how the associated sequence of texture-induced whisker vibrations is further encoded by the CNS, by focusing on the barrel cortex. The mechanical vibrations and electronic noise induced by the motorized stage hampered neurophysiological recordings during actual texture-stimulation experiments. Texture-induced whisker movements, optically extracted from an actual experiment, were thus replayed on 24 whiskers on one side of the animal snout using a custom-made whisker stimulator (Jacob et al., 2010).

Figure $8 \mathrm{~A}$ shows a raster plot of a single-unit response recorded in the $\mathrm{S} 1$ area. The discharge rate of the cell closely follows the envelope $A(t)$ of the imposed whisker oscillations (Fig. $8 B$ ). This observation was made across single and multi-units, independently of the mean firing rate of the recorded unit (Fig. 8C). To quantify this observation, the maximum of the crosscorrelation between $A(t)$ and the cortical firing rate was compared with a previously computed threshold of significance (see Materials and Methods). When performed throughout all recordings, this analysis showed that the response of $26 \%$ of single units (SU; 45/170) and of $44 \%$ of multi-units (MU; 157/344) is driven by the stimulus envelope.

The firing rate varies quasi-linearly with the stimulus envelope $A(t)$ beyond a finite threshold as shown in Figure $9 A$. This observation holds true for both single units $\left(R^{2}=0.71 \pm 0.03\right.$ (SEM); $N=45)$ and multi-units $\left(R^{2}=0.72 \pm 0.01(\mathrm{SEM}) ; N=157\right)$ recordings. Notice that the discharge rate measured below the threshold stimulus amplitude is similar to the spontaneous discharge rate of the neurons as evaluated during interstimulus periods (SU: $R^{2}=0.66$; MU: $R^{2}=0.67$ ).

The threshold amplitude is $42.3 \pm 2.1 \mu \mathrm{m}$ (SEM) for single units and $43.5 \pm 0.9 \mu \mathrm{m}$ (SEM) for multi-units (Fig. 9B), respectively. The difference between these two values is not statistically significant ( $p=0.73$ ). We also observed that the $A$ versus firing rate curve is consistent between single and multi-units. The slope of this curve is $0.25 \pm 0.05 \mathrm{AP} \cdot \mathrm{s}^{-1} \cdot \mu \mathrm{m}^{-1}$ (SEM) for single units and $0.25 \pm 0.02 \mathrm{AP} \cdot \mathrm{s}^{-1} \cdot \mu \mathrm{m}^{-1}(\mathrm{SEM})$ for multi-units (Fig. 9C). Again, the difference between these two values is unsignificant $(p=0.06)$.

The observed linear dependence of the spiking rate with the stimulus vibratory amplitude is consistent with previous experiments in which the spiking rate was found to be linearly driven by the whisker mean velocity (Arabzadeh et al., 2003; Adibi et al., 2012). In the present case, the replayed stimulus was dominated 

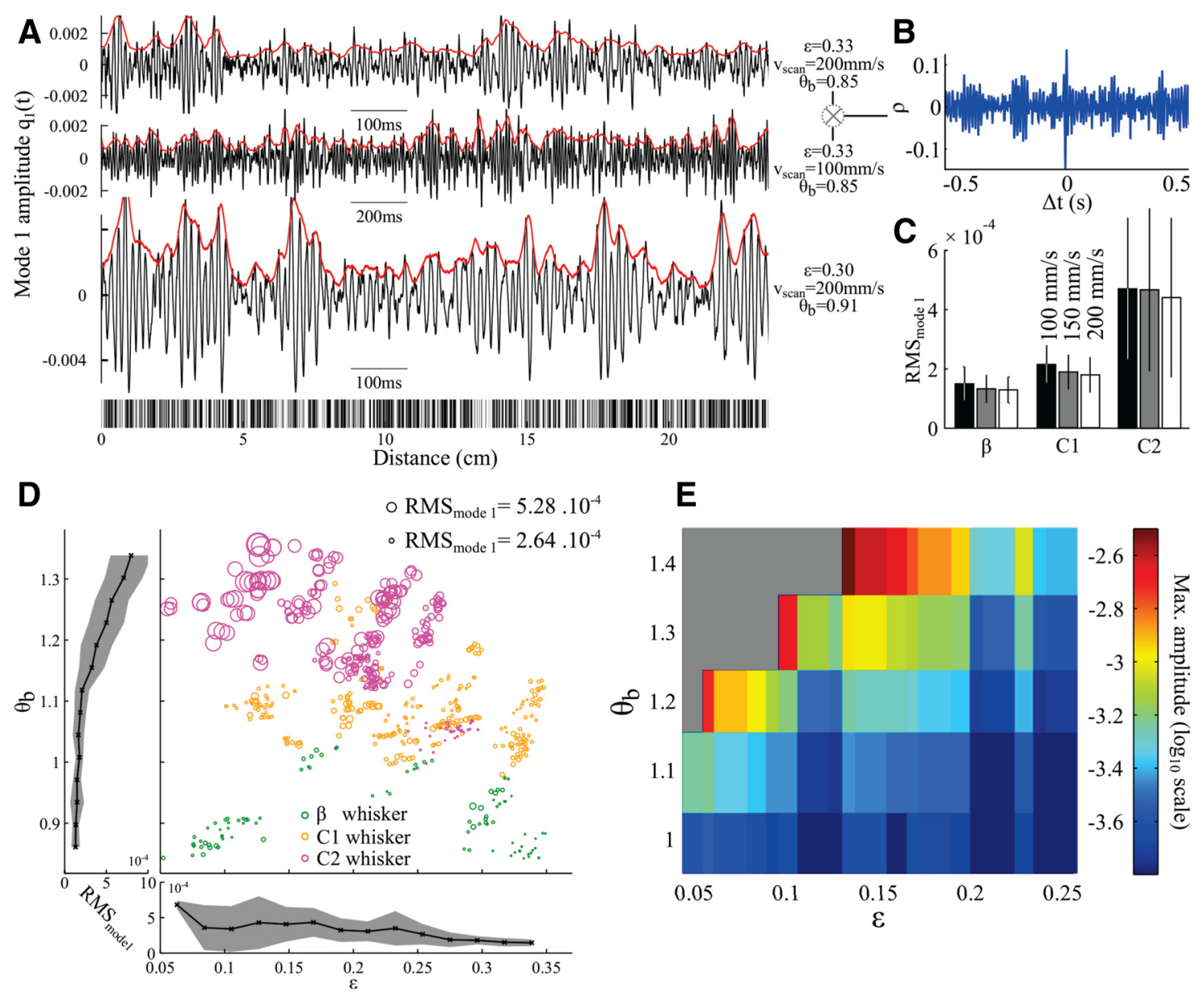

Figure 7. Effect of the exploratory parameters on the transduction gain. $A$, Time series of the first mode amplitude for two contact point locations and two scanning speeds (envelopes are shown in red). The explored topography for the three series is shown below. Resonance frequencies of the three signals (top to bottom): 88,69 , and $89 \mathrm{~Hz}$. $\boldsymbol{B}$, Cross-correlograms between sweeps performed at two different scanning speeds. $\boldsymbol{C}$, Measured RMS mode $1_{1}$ for different whiskers and scanning speeds in the experimental data. $\boldsymbol{D}$, Dependence of the first mode rms on the base angle $\theta_{b}$ and contact point position $\varepsilon$ (experiments). The radius of each circle is proportional to the first mode rms. Mean $\mathrm{RMS}_{\text {mode } 1}$ across all data $=2.64 .10^{-3} \cdot \mathbf{E}$, Maximal normal displacement in $s=0.9$ (see inset in Fig. 6A) for varying $\theta_{b}, \varepsilon(\lambda=1 \mathrm{~mm})$ (model). The gray zone indicates the region where the calculation could not be performed (see Materials and Methods).

by the fundamental resonant mode such that the whisker velocity can be well approximated by $A(t) \omega$. As the resonant frequency $\omega$ remains constant during a sweep, the whisker velocity, and thus the neuronal spiking rate, directly encode the whisker resonant amplitude $A(t)$.

\section{Discussion}

The present study aimed at providing a comprehensive description of the process of texture transduction at play during tactile exploration in whisking rodents, from the whisker tip to the somatosensory cortex. Our findings suggest that fine textural features are mechanically encoded by the whiskers through a vibratory amplitude modulation scheme. As the whisker tip is scanned across the probed surface, the texture-evoked whisker vibrations are dominated by the fundamental resonant mode and can thus be correctly approximated by $A(t) \cos (\omega t+\phi(t))$. In this expression, the carrier-wave frequency $\omega$, set by the fundamental resonant frequency of the contacting whisker, does not depend on the surface texture. Textural information is in turn entirely contained in the slowly varying envelope $A(t)$. This mechanical signal, which drives the response of the peripheral receptors located in the follicle, is further demodulated along the somatosensory pathway, yielding a direct spike-rate encoding of the amplitude $A(t)$ in the $\mathrm{S} 1$ region.
This scenario for texture transduction has several interesting properties. As established by the present work, the carrier-wave frequency depends neither on the scanning speed nor on the whisker base angle, and is weakly dependent on the pad-tosurface distance. In most ethological conditions, the dominant frequency of the whisker vibrations should thus vary within a relatively narrow range, thus facilitating the detection of small signals (associated with fine textural elements) by frequencytuned mechanoreceptors (Arabzadeh et al., 2005). The envelope signal itself appears to weakly vary with both the scanning speed and pad-to-surface distance. The characteristics of the cortical signature elicited by a particular surface topography should thus be relatively robust with regard to variations in exploratory conditions, thus enhancing texture discrimination performances. Notice, however, that the envelope amplitude can be actively tuned by the animal through adjustment of the whisker base angle relative to the probed surface.

The proposed encoding scheme offers the possibility for a parallel transduction of large- and fine-scale textural properties through independent sensory channels. As demonstrated in this study, resonant whisker oscillations are principally elicited by discrete gap-crossing events. Such processes become irrelevant for surface corrugations whose curvatures fall below that of the 


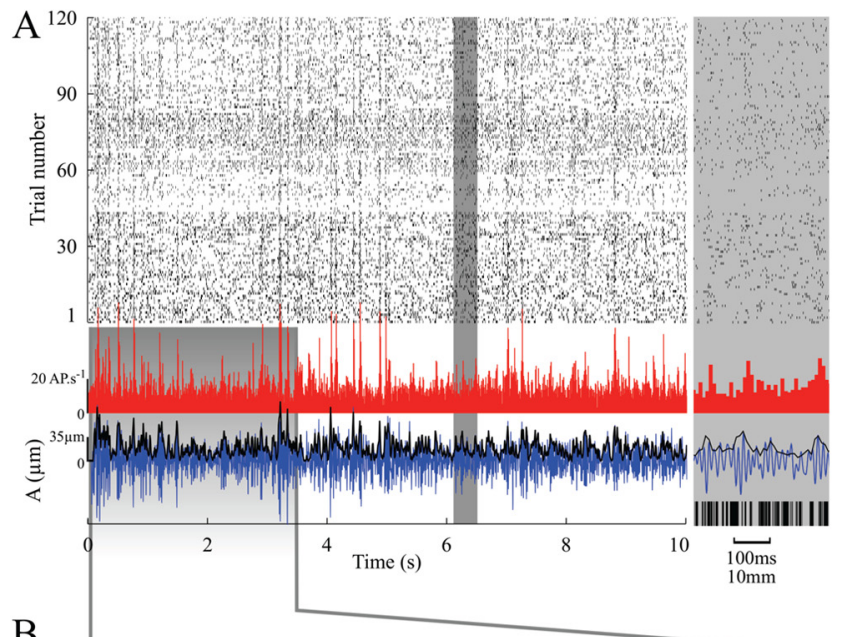

B
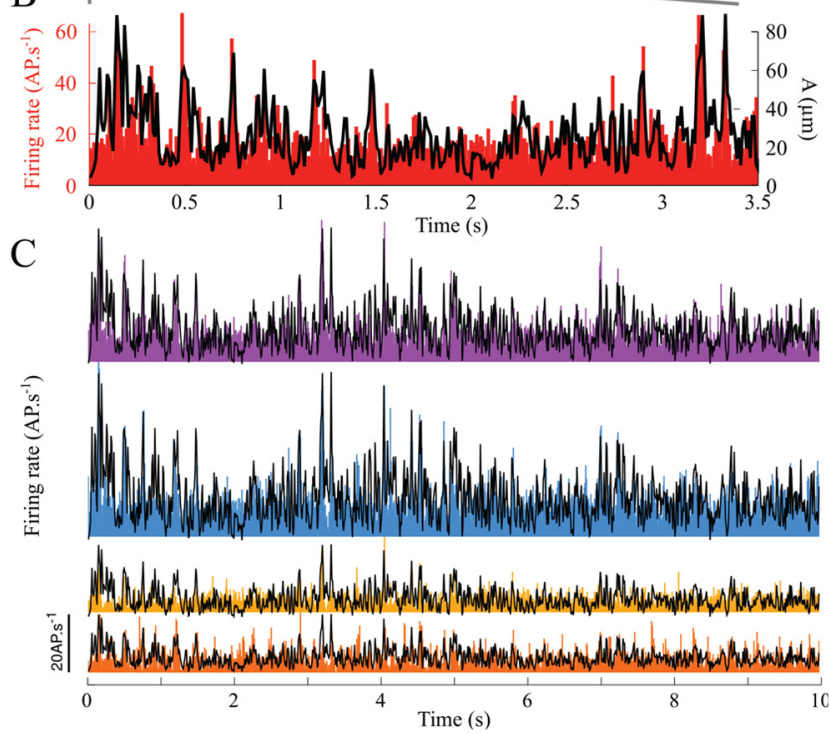

Figure 8. Cortical response to texture-induced whisker oscillations. $A$, Single-unit activity elicited by texture-induced whisker movements replayed using a multiwhisker stimulator. Left, From top to bottom: raster plot (120 trials), firing rate (red histogram, bin size $=10 \mathrm{~ms}$ ), replayed stimulus on the principal whisker (blue line), and envelope $A$ (black line). Right, Zoom of the shaded region on the left. The corresponding portion of the texture topography is displayed below. $\boldsymbol{B}$, The neuronal firing rate (red histogram) follows the envelope amplitude $A$ of the whisker movement (black line; bin size $=10 \mathrm{~ms}$ ). C, Average discharge rate for two multiunits (cool colors) and two single units (warm colors) simultaneously recorded are shown with the stimulus envelope $A$ (black lines). A scaling coefficient was applied to $A$ to best match the neuronal firing rate amplitude.

whisker tip (of the order of $1 \mathrm{~cm}^{-1}$ ). Such large-scale surface modulations will, however, drive a low-frequency force and moment signal at the follicle (Schroeder and Hartmann, 2012), which can be registered by slowly adapting mechanoreceptors. These slowly varying baseline modulations will marginally impact the resonant dynamics of the whisker, whose amplitudes are controlled by the fine-scale textural properties and are encoded by rapidly adapting mechanoreceptors. This parallel processing mechanism may thus allow the animal to efficiently identify textures on nonplanar surfaces, while simultaneously extracting information about the large-scale shape of the probed object.

This dual-channel encoding scheme for fine and coarse features is strongly reminiscent of the so-called duplex theory for texture perception in human digital touch. In this sensory system, large features (in the millimeter scale and above) are encoded by populations of slowly adapting cutaneous receptors (Merkel

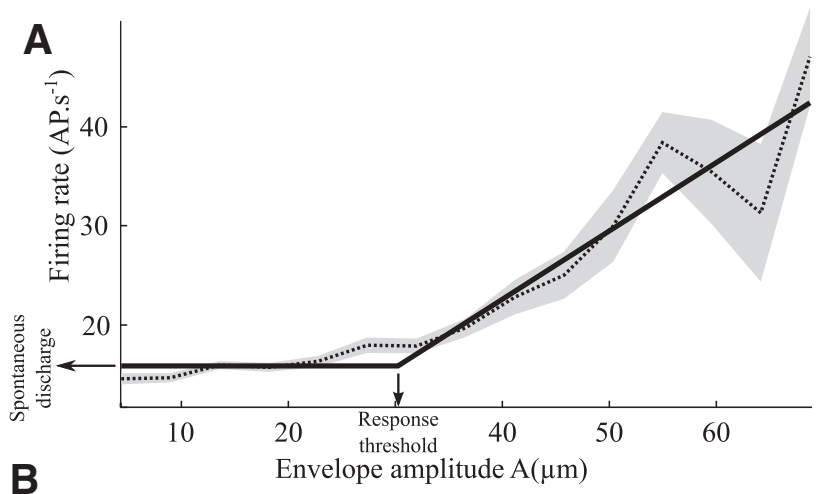

B
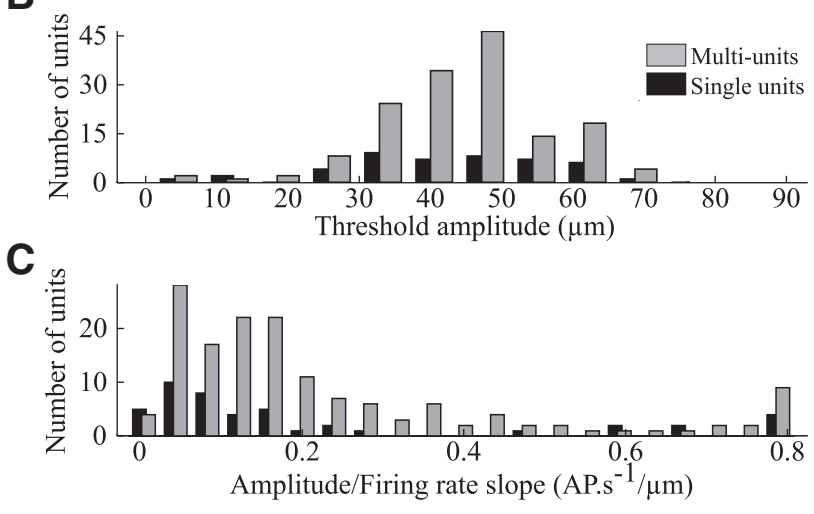

Figure 9. Dependence of the neuronal firing rate with the stimulus envelope. $\boldsymbol{A}$, The firing rate displays a linear dependence with the stimulus amplitude beyond a threshold value, as illustrated by the two-segment fit. $\boldsymbol{B}$, Distribution of the envelope amplitude threshold measured for single and multi-units that displayed texture-modulated firing rate. C, Distribution of the slope of the linear relationship between the envelope amplitude $A$ and the neuronal firing rate for the same units.

cells), while fine-scale features are mediated by rapidly adapting receptors (Pacinian corpuscles). It has recently been suggested that this second channel of perception may benefit from two non-neural processes that both selectively amplify skin vibrations within the optimal spectral range of the Pacinian receptors. One results from the presence of epidermal ridges at the surface of the skin (Scheibert et al., 2009), and the second is associated with the intrinsic resonance properties of the digital skin (Manfredi et al., 2012) and is thus analogous to the mechanism described in the present study. As in the whisker system, skin vibratory amplitude appears to be encoded in the strength of the response evoked in S1 neurons as evidenced in awake macaques (Harvey et al., 2013).

Building on previous descriptions of the whisker biomechanics, a comprehensive model of texture transduction was developed that correctly captures the spectral and temporal characteristics of rapid minute oscillations of macrowhiskers induced upon scanning micropatterned substrates. It provides the first predictive model of texture-to-whisker transduction. A major outcome of this model is to clarify the way the transduction process depends on exploratory conditions. It also provides a consistent interpretation of how texture transduction operates at the microscopic frictional contact between the whisker tip and the substrate, an aspect that has been ignored in earlier models. Two distinct driving mechanisms could be identified. The first one, associated with the continuous displacement imposed to the tip of the whisker as it is scanned across the surface topography, elicits whisker oscillations whose amplitude varies linearly with the whisker scanning speed (see Eq. 12). The second process is associated with brief, stick-slip like events that occur as the whisker suddenly changes 
conformation following a gap-crossing event. The oscillations triggered by this second mechanism are expected to be essentially insensitive to the scanning speed, and have an amplitude proportional to the gap width (see Eq. 13). The comparison of the two equations indicates that this second process should be dominant whenever $\lambda \omega / v_{\text {scan }}>1$, which appears to be the case in ethological conditions. This result thus rationalizes the previously proposed kinetic signature model, in which stick-slip-induced oscillations were hypothesized to carry the most significant signal (Arabzadeh et al., 2005; Wolfe et al., 2008). The present analysis demonstrates that (1) these events are ubiquitous during texture exploration phases, (2) each of them triggers a bout of whisker oscillations whose amplitude directly reflects the in-plane size of the textural features, and (3) they cumulatively drive the whisker resonance envelope in a texture-specific way.

Refinements of the model are in order. First, the current theoretical description of whisker resonance is strictly valid in the limit of small deformations. Although this approximation allowed us to correctly capture the resonant frequencies, corrective terms may still be significant for largely deformed whiskers. Second, the model does not account for intrinsic whisker-specific curvature that may modify the contact configuration and hence impact the transduction process. More importantly, the present work addressed a rather simple configuration in which the whiskers were passively dragged along a surface near-parallel to the animal anteroposterior axis, while tactile exploration generally involves active whisking onto an object located in front of the animal's snout. Such configurations involve complex 3D whisker dynamics (Knutsen et al., 2008) during which the base angle $\theta_{b}$ and the contact position $\varepsilon$ continuously vary. The $\theta_{b}$ variations might be accounted for in a straightforward way by using a continuously varying gain factor. The change in contact location along the whisker shaft might be more problematic. Indeed, this quantity controls both the gain and the effective resonance frequency of the contacting whisker. However, these dependences being relatively weak, considering a single (mean) value for this parameter should yield a reasonable approximation of the biomechanical transduction process.

Numerous questions remain regarding the neural mechanism underlying the demodulation process taking place between the whisker follicle and the somatosensory cortex. One may also wonder about the relevant metrics in the spiking signal of the cortical neurons that control the animal's ability to distinguish between two different textures in forced choice experiments. One possibility would be that the discrimination relies on the mean spike rate. Another option, which would probably provide a better efficiency, would rely on the first moment of the spike rate distribution, i.e., a measure of the time-fluctuating nature of the cortical response. More experiments are needed, that would combine electrophysiology and behavioral assays, to conclude on these aspects.

\section{References}

Adibi M, Diamond ME, Arabzadeh E (2012) Behavioral study of whiskermediated vibration sensation in rats. Proc Natl Acad Sci U S A 109:971976. CrossRef Medline

Arabzadeh E, Petersen RS, Diamond ME (2003) Encoding of whisker vibration by rat barrel cortex neurons: implications for texture discrimination. J Neurosci 23:9146-9154. Medline
Arabzadeh E, Zorzin E, Diamond ME (2005) Neuronal encoding of texture in the whisker sensory pathway. PLoS Biol 3:e17. CrossRef Medline

Birdwell JA, Solomon JH, Thajchayapong M, Taylor MA, Cheely M, Towal RB, Conradt J, Hartmann MJ (2007) Biomechanical models for radial distance determination by the rat vibrissal system. J Neurophysiol 98: 2439-2455. CrossRef Medline

Boubenec Y, Shulz DE, Debrégeas G (2012) Whisker encoding of mechanical events during active tactile exploration. Front Behav Neurosci 6:74. CrossRef Medline

Hartmann MJ, Johnson NJ, Towal RB, Assad C (2003) Mechanical characteristics of rat vibrissae: resonant frequencies and damping in isolated whiskers and in the awake behaving animal. J Neurosci 23:6510-6519. Medline

Harvey MA, Saal HP, Dammann JF 3rd, Bensmaia SJ (2013) Multiplexing stimulus information through rate and temporal codes in primate somatosensory cortex. PLoS Biol 11:e1001558. CrossRef Medline

Hill DN, Curtis JC, Moore JD, Kleinfeld D (2011) Primary motor cortex reports efferent control of vibrissa motion on multiple timescales. Neuron 72:344-356. CrossRef Medline

Jacob V, Estebanez L, Le Cam J, Tiercelin JY, Parra P, Parésys G, Shulz DE (2010) The matrix: a new tool for probing the whisker-to-barrel system with natural stimuli. J Neurosci Methods 189:65-74. CrossRef Medline

Jenks RA, Vaziri A, Boloori AR, Stanley GB (2010) Self-motion and the shaping of sensory signals. J Neurophysiol 103:2195-2207. CrossRef Medline

Knutsen PM, Biess A, Ahissar E (2008) Vibrissal kinematics in 3D: tight coupling of azimuth, elevation, and torsion across different whisking modes. Neuron 59:35-42. CrossRef Medline

Lottem E, Azouz R (2008) Dynamic translation of surface coarseness into whisker vibrations. J Neurophysiol 100:2852-2865. CrossRef Medline

Lottem E, Azouz R (2009) Mechanisms of tactile information transmission through whisker vibrations. J Neurosci 29:11686-11697. CrossRef Medline

Manfredi LR, Baker AT, Elias DO, Dammann JF 3rd, Zielinski MC, Polashock VS, Bensmaia SJ (2012) The effect of surface wave propagation on neural responses to vibration in primate glabrous skin. PLoS One 7:e31203. CrossRef Medline

Morita T, Kang H, Wolfe J, Jadhav SP, Feldman DE (2011) Psychometric curve and behavioral strategies for whisker-based texture discrimination in rats. PLoS One 6:e20437. CrossRef Medline

Neimark MA, Andermann ML, Hopfield JJ, Moore CI (2003) Vibrissa resonance as a transduction mechanism for tactile encoding. J Neurosci 23:6499-6509. Medline

Persson BNJ (2000) Sliding friction: physical principles and applications. Berlin: Springer.

Quist BW, Faruqi RA, Hartmann MJ (2011) Variation in young's modulus along the length of a rat vibrissa. J Biomech 44:2775-2781. CrossRef Medline

Ritt JT, Andermann ML, Moore CI (2008) Embodied information processing: vibrissa mechanics and texture features shape micromotions in actively sensing rats. Neuron 57:599-613. CrossRef Medline

Scheibert J, Leurent S, Prevost A, Debrégeas G (2009) The role of fingerprints in the coding of tactile information probed with a biomimetic sensor. Science 323:1503-1506. CrossRef Medline

Schroeder CL, Hartmann MJ (2012) Sensory prediction on a whiskered robot: a tactile analogy to "optic flow." Front Neurorobot 6:9. CrossRef Medline

Solomon JH, Hartmann MJ (2006) Biomechanics: robotic whiskers used to sense features. Nature 443:525. CrossRef Medline

Whishaw IQ, Kolb B (2005) The behaviour of the laboratory rat. Oxford, UK: Oxford UP.

Williams CM, Kramer EM (2010) The advantages of a tapered whisker. PLoS One 5:e8806. CrossRef Medline

Wolfe J, Hill DN, Pahlavan S, Drew PJ, Kleinfeld D, Feldman DE (2008) Texture coding in the rat whisker system: slip-stick versus differential resonance. PLoS Biol 6:e215. CrossRef Medline 